CERN-PH-TH/2013-108

SISSA 23/2013/FISI

\title{
The Role of Electroweak Corrections for the Dark Matter Relic Abundance
}

\author{
Paolo Ciafaloni ${ }^{\text {a }}$, Denis Comelli ${ }^{\text {b }}$, Andrea De Simone ${ }^{c, d, e}$, \\ Enrico Morgante ${ }^{f}$, Antonio Riotto $^{f}$, Alfredo Urbano $^{d}$ \\ a Dipartimento di Fisica, Università di Lecce and INFN - Sezione di Lecce, \\ Via per Arnesano, I-73100 Lecce, Italy \\ b INFN - Sezione di Ferrara, Via Saragat 3, I-44100 Ferrara, Italy \\ c CERN, PH-TH Division, CH-1211, Genève 23, Switzerland \\ d SISSA, Via Bonomea 265, I-34136 Trieste, Italy \\ e INFN, Sezione di Trieste, I-34136 Trieste, Italy \\ f Département de Physique Théorique and Centre for Astroparticle Physics (CAP), \\ 24 quai E. Ansermet, CH-1211 Genève, Switzerland
}

\begin{abstract}
We analyze the validity of the theorems concerning the cancellation of the infrared and collinar divergences in the case of dark matter freeze-out in the early universe. In particular, we compute the electroweak logarithmic corrections of infrared origin to the annihilation cross section of a dark matter particle being the neutral component of a $\mathrm{SU}(2)_{L}$ multiplet. The inclusion of processes with final state $W$ can modify significantly the cross sections computed with only virtual $W$ exchange. Our results show that the inclusion of infrared logs is necessary for a precise computation of the dark matter relic abundance.
\end{abstract}




\section{Introduction}

The abundance of cold Dark Matter (DM) in our universe is now experimentally known with a great precision, $\Omega_{\mathrm{DM}} h^{2}=0.1199 \pm 0.0027$, thanks to the Planck satellite recent data [1]. Very significant and diverse experimental efforts are currently ongoing to identify the nature and the interactions of the DM particle, other than the gravitational ones, but in spite of this, a convincing signal is still missing.

A great deal of attention has been recently devoted to the study of the effects of ElectroWeak (EW) radiation, especially about their impact on the predictions for indirect DM searches [2-14] (for earlier studies on the impact of gauge boson radiation on DM annihilations or cosmic ray physics, see $[15-17])$.

In this paper, we study the effects of EW corrections for DM physics in a different setup, namely the epoch of DM freeze-out in the early universe, when the DM relic density is established. In view of the precision on the relic density measurements, several studies have been performed to refine the theoretical calculations at a level comparable with the experimental uncertainties. For instance, the radiative one-loop corrections to the DM annihilation cross section have been computed, especially in the context of supersymmetric DM (see e.g. Refs. [18-22]). These studies are particularly important in view of the fact that thermally averaged cross section required to reproduce the relic DM abundance can be calculated with a precision smaller than a few percent, once the relatively rapid changes (especially during the EW and QCD phase transitions) in the number of degrees of freedom with the temperature is properly accounted for [23].

The radiation of EW gauge bosons in the annihilation process are typically not considered, relying on the cancellation of infrared and collinear divergences, after a suitable average over the initial and final states is performed. What we point out in this paper is that the conditions of validity of the cancellation theorems are not satisfied if the DM particle belongs to an $\mathrm{SU}(2)_{L}$ multiplet, whose components are not degenerate in mass. In such a situation, we find potentially large corrections of infrared origin affecting the calculation of the annihilation cross sections, and hence the predictions for the DM relic abundance.

The paper is organized as follows. In Section 2 we review the cancellation theorems, their violations and we study the case of DM freeze-out. In Section 3 we present an explicit calculation of the infrared logs, and show its relevance. Finally, we summarize our main results and draw our conclusions in Section 4. In Appendix A we explicitly work out the cancellation of collinearly divergent logarithms, to show how the cancellation theorems work in the case of degenerate states.

\section{The violation of the Bloch-Nordsieck theorem for the DM freeze- out}

\subsection{The cancellation theorems and their violations}

The Kinoshita-Lee-Nauenberg (KLN) theorem [24] states that in a field theory with massless fields the cross sections are free of both soft and collinear divergences if summed over both final and initial degenerate states. Here degenerate states are meant to be degenerate in energy (infrared) and angle (collinear) up to the resolving power of any given experiment. In fact, this cancellation theorem for the InfraRed (IR) divergences in QED dates back to the pioneering work of Bloch and 
Nordsieck (BN) [25] (see also [26] for the coherent state approach) and successively extended to non abelian field theories [27]. The IR and collinear divergences manifest themselves as single or double logarithms of the ratio of the energy scale involved in the hard process over an IR scale and hence they can generate large radiative corrections.

We will show that potential large logs contributing to DM annihilation processes are partially cancelled only once one defines appropriate averaged cross sections. The precise relation between thermally-averaged cross sections and cancellation theorems is our main result.

Let us assume that the DM particle is the electrically neutral Majorana component of a $\mathrm{SU}(2)_{L}$ multiplet $\chi^{a}$ with hypercharge $Y=0$ (for example, the case of a triplet can be the wino in supersymmetry). Consider now the annihilation processes of the type $\chi^{a} \chi^{b} \rightarrow f, \chi^{a} \chi^{b} \rightarrow f+W_{f}$ (emission) and $\chi^{a} \chi^{b}+W_{i} \rightarrow f$ (absorption), where $f$ stands for a two-particle final state, typically made out of two fermions. Schematically, the KLN theorem states that the sum

$$
\sum_{a, b} \sum_{i, f}\left[\left|\mathcal{M}^{(0)}+\mathcal{M}^{(1)}\right|_{a b \rightarrow f}^{2}+\int\left(\mathrm{d} \Pi_{W_{f}}\right)|\mathcal{M}|_{a b \rightarrow f+W_{f}}^{2}+\int\left(\mathrm{d} \Pi_{W_{i}}\right)|\mathcal{M}|_{a b+W_{i} \rightarrow f}^{2}\right]
$$

is free of IR and collinear logarithmic divergences. Here $\mathcal{M}^{(0)}$ and $\mathcal{M}^{(1)}$ are the tree-level and oneloop amplitude of the process $\chi^{a} \chi^{b} \rightarrow f$, respectively, and $\mathrm{d} \Pi_{W_{i, f}}$ indicate the integral measures for the absorbed and emitted gauge boson, respectively. In other words, the IR and collinear logarithmic divergences disappear upon averaging over the initial flavor $a$ and the initial degenerate $W_{i}$ 's in the initial states, and summing over the final flavor and the final degenerate $W_{f}$ 's in the final states. On the other hand, the BN theorem $[27]^{1}$ states that the sum

$$
\sum_{a, b} \sum_{f}\left[\left|\mathcal{M}^{(0)}+\mathcal{M}^{(1)}\right|_{a b \rightarrow f}^{2}+\int\left(\mathrm{d} \Pi_{W_{f}}\right)|\mathcal{M}|_{a b \rightarrow f+W_{f}}^{2}\right]
$$

is also free of IR divergences when one averages over the initial flavors $a$ in the initial states and sums over the final flavor and the final degenerate $W_{f}$ 's in the final states.

If some of the hypotheses of these cancellation theorems does not hold, their conclusions are evaded and in general single or double logs (of infrared/collinear origin) will appear in the calculation of the annihilation cross section. Therefore, when the cancellation theorems are not operative, one expects corrections (at least) of the order

$$
\mathcal{O}\left(\mathcal{N} \cdot \frac{\alpha_{W}}{4 \pi} \cdot \ln ^{n} \frac{M_{1}^{2}}{m_{W}^{2}}\right), \quad n=1,2,
$$

where $\mathcal{N}$ is a numerical coefficient (typically large, as discussed later), $\alpha_{W} \equiv g^{2} / 4 \pi \approx 0.03$ and $M_{1}$ indicates the DM mass, assumed to be much larger than the weak scale $M_{1} \gg m_{W}$. Therefore, these corrections can be large and even reach the order of $100 \%$. So, the crucial question is: under which circumstances are the cancellation theorems not operative?

One case in which this happens is at the LHC [29]. Indeed, since the colliding initial states carry a definite non-abelian flavor, EW radiative corrections to inclusive hard cross sections at the $\mathrm{TeV}$ scale are affected by peculiar BN-violating logs $[29,30]$. In fact, in such a case, the corrections are

\footnotetext{
${ }^{1}$ The above cancellation mechanism was shown to operate in the context of exact non-abelian gauge theories and later extended to the spontaneously broken abelian [28] and non abelian field theories [29], where different levels of inclusiveness are possible.
} 
even the larger one in the estimate (2.3) because the system is highly relativistic and both collinear and IR divergences are present, leading to double-log $\ln ^{2}\left(M^{2} / m_{W}^{2}\right)$ enhancement.

Another case where large EW logs enter is in indirect searches for DM, where the relevant quantities are the energy spectra of final state particles originating from the annihilation of DM in the Milky Way. These are partially inclusive quantities, as the energy spectra do not involve integration over the full phase space of final particles, so the total inclusiveness requested by the cancellation theorems is not respected. Therefore, the final fluxes of stable particles originated by DM annihilations are strongly affected by the real and virtual emission of $W$ gauge bosons.

Finally, yet another situation where the cancellation theorems fail to screen the logarithmic corrections is when the initial states are not degenerate in mass. In this case, the average over the initial flavor upsets the delicate cancellations of logarithms at work when the initial state is completely mass-degenerate. An example of this situation is the annihilation of DM particles, belonging to a $\mathrm{SU}(2)_{L}$ multiplet, whose components are not degenerate in mass. In the early universe, around the epoch of the freeze-out of the DM particles when their relic abundance is established, the violation of the cancellation theorems is also of dynamical type. In order to make the theorems operative, one should sum over the initial states. However, they can be non-degenerate in mass and so differently populated in the thermal plasma and differently weighted by an exponential Boltzmann factor $\exp \left(-M_{a} / T\right)$, where $M_{a} \sim M_{1}$ is the mass of the state $\chi^{a}$ and $T \sim M_{1} / 25$ is the temperature of the universe around the freeze-out. Conversely, only in the limit where all the particles of the multiplet which DM belongs to are exacly degenerate in mass, the cancellation theorems apply and the correction (2.3) is not present. The study of this situation is the central topic of the present paper.

\subsection{EW corrections for the DM freeze-out}

Let us elaborate further about the violation of the cancellation theorems in presence of nondegenerate DM multiplets and the impact this has on the relic density calculation. For definiteness, let us assume that the DM particle is the electrically neutral Majorana component of a $\mathrm{SU}(2)_{L}$ triplet $\chi^{a}(a=1,2,3)$ with hypercharge $Y=0$. We will work out this example explicitly, but the conclusions are general and can be translated straighforwardly to any model where the DM is part of an $\mathrm{SU}(2)_{L}$ multiplet. In order to capture the impact of the cancellation theorems in a model-independent way, we work with an effective field theory Lagrangian, restricting ourselves to interactions of the DM triplet with the SM left-handed doublet $L=\left(f_{1}, f_{2}\right)^{T}$. The most general dimension-six operators are

$$
\mathscr{L}_{\mathrm{eff}}=\frac{C_{\mathrm{D}}}{\Lambda^{2}} \delta_{a b}\left(\bar{L} \gamma_{\mu} P_{L} L\right)\left(\bar{\chi}^{a} \gamma^{\mu} \gamma_{5} \chi^{b}\right)+i \frac{C_{\mathrm{ND}}}{\Lambda^{2}} \epsilon_{a b c}\left(\bar{L} \gamma_{\mu} P_{L} \sigma^{c} L\right)\left(\bar{\chi}^{a} \gamma^{\mu} \chi^{b}\right)
$$

where $C_{\mathrm{D}}$ and $C_{\mathrm{ND}}$ are real coefficients for diagonal and non-diagonal interactions in isospin space, $P_{R, L}=\left(1 \pm \gamma_{5}\right) / 2$ and $\sigma^{c}$ are the Pauli matrices.

Let us consider the Boltzmann equation describing the number density $n=\sum_{a} n_{a}$ of the particles 
$\chi^{a}$ in the plasma

$$
\begin{aligned}
a^{-3} \frac{\mathrm{d}\left(n a^{3}\right)}{\mathrm{d} t}= & -\sum_{a, b, f}\left\langle v \sigma_{a b \rightarrow f}\right\rangle\left(n_{a} n_{b}-n_{a}^{\mathrm{eq}} n_{b}^{\mathrm{eq}}\right) \\
& -\sum_{a, b, f}\left\langle v \sigma_{a b \rightarrow f+W_{f}}\right\rangle\left(n_{a} n_{b}-n_{a}^{\mathrm{eq}} n_{b}^{\mathrm{eq}} \frac{n_{W_{f}}}{n_{W_{f}}^{\mathrm{eq}}}\right) \\
= & -\sum_{a, b, f}\left(\left\langle v \sigma_{a b \rightarrow f}\right\rangle+\left\langle v \sigma_{a b \rightarrow f+W_{f}}\right\rangle\right)\left(n_{a} n_{b}-n_{a}^{\mathrm{eq}} n_{b}^{\mathrm{eq}}\right),
\end{aligned}
$$

where in the last passage we have assumed that the gauge bosons are in kinetic equilibrium and we have disregarded the contribution from the absorption of the $W_{i}$, which in the Boltzmann equation is exponentially suppressed compared to the other terms. This is true if we work in the regime $T \sim M_{1} / 25 \lesssim m_{W}$, i.e. as long as the $\mathrm{DM}$ is lighter than a few $\mathrm{TeV}$. Under this approximation of neglecting the absorption of $W$ from the initial state, the cancellation theorem possibly at work here is the BN theorem, and evading this theorem would give rise to IR logs.

Upon introducing the quantities [31]

$$
r_{a}=\frac{n_{a}^{\mathrm{eq}}}{n^{\mathrm{eq}}}=\frac{g_{a}\left(1+\Delta_{a}\right)^{3 / 2} e^{-x \Delta_{a}}}{g_{\mathrm{eff}}}
$$

where $x=M_{1} / T, \Delta_{a}=\left(M_{a}-M_{1}\right) / M_{1}$ and $g_{\text {eff }}=\sum_{a} g_{a}\left(1+\Delta_{a}\right)^{3 / 2} \exp \left(-x \Delta_{a}\right)$, one can rewrite the Eq. (2.5) as

$$
a^{-3} \frac{\mathrm{d}\left(n a^{3}\right)}{\mathrm{d} t}=-\left\langle v \sigma_{\text {eff }}\right\rangle\left(n^{2}-n_{\text {eq }}^{2}\right)
$$

where

$$
\sigma_{\mathrm{eff}}=\sum_{a, b, f} r_{a} r_{b}\left(\sigma_{a b \rightarrow f}+\sigma_{a b \rightarrow f+W_{f}}\right)
$$

This object almost contains the expression (2.2), with the crucial difference of the weights $r_{a}$

$$
\sum_{a, b} \sum_{f} r_{a} r_{b}\left[\left|\mathcal{M}^{(0)}+\mathcal{M}^{(1)}\right|_{a b \rightarrow f}^{2}+\int\left(\mathrm{d} \Pi_{W_{f}}\right)|\mathcal{M}|_{a b \rightarrow f+W_{f}}^{2}\right] .
$$

An exactly mass-degenerate DM multiplet would have the same $r_{a}$, for all $a$, and the expression (2.2) would be recovered. Instead, if the masses of the $\chi^{a}$ particles are different from each other, the $\mathrm{BN}$ theorem is not operative. In particular, if the mass splitting

$$
\Delta_{a} \gtrsim \frac{1}{25}
$$

(where $x_{f}=M_{1} / T_{f} \simeq 25$ and $e^{-x_{f} \Delta_{a}} \leq 1$ ) we expect logarithmic divergences of the form (2.3) to appear in the computation of the final abundance. Notice that in the cosmological setup only single logs of IR origin appear, as the initial states are slightly non-relativistic. The reader can find more details about this point in the Appendix A.

Conversely, in the regime of efficient annihilation $\Delta_{a} \lesssim 1 / 25$ (where $e^{-x_{f} \Delta_{a}} \sim 1-x_{f} \Delta_{a}$ ), we expect that the common computation of the freeze-out abundance of DM is correct up to corrections at most of the order

$$
\mathcal{O}\left(25 \cdot \Delta_{a} \cdot \mathcal{N} \cdot \frac{\alpha_{W}}{4 \pi} \cdot \ln \frac{M_{1}^{2}}{m_{W}^{2}}\right)
$$




\begin{tabular}{||c|c||}
\hline Cross Section & Expected Corrections \\
\hline \hline $2 \rightarrow 2$ with 1-loop radiative corrections & $\alpha_{W} \ln \frac{M_{1}^{2}}{m_{W}^{2}}, \alpha_{W} \ln ^{2} \frac{M_{1}^{2}}{m_{W}^{2}}$ \\
\hline $2 \rightarrow 2+W_{f}$, no average over initial flavor & $\alpha_{W} \ln \frac{M_{1}^{2}}{m_{W}^{2}}$ \\
\hline $2 \rightarrow 2+W_{f}$, average over initial non-degen. flavor & $\alpha_{W} \Delta_{a} \ln \frac{M_{1}^{2}}{m_{W}^{2}}$ \\
\hline
\end{tabular}

Table 1: EW IR corrections involved into the evaluation of the various cross sections depending on the degree of inclusiveness. The first line refers to the usual Sudakov logs appearing when virtual corrections are included. The correction in the second line appears when no average over the initial state is performed, and BN theorem is evaded. The last correction originates from for the dynamical violation of the IR cancellation provided by the BN theorem in thermal averaged cross sections, in presence of non-degenerate initial states.

The $2 \rightarrow 2$ cross sections include the EW one loop radiative corrections and are affected by Sudakov form factor corrections of single and double log size coming mainly from the final state virtual corrections [32]. The leading double-log corrections are then cancelled by the inclusion of real $W$ final states that reduce the degree of singularity to single-log factors whose origin can be identified with a violation of the $\mathrm{BN}$ theorem [29] and the fact that the initial states are partially nonrelativistic. Finally, the fact that the DM multiplet contains particles almost mass-degenerate during the cohannilation processes partially screens the single logs by a further coefficient $\Delta_{a}$ [33]. In Table 1 we give schematically the order of magnitude of the IR corrections expected once we compute different kinds of cross sections involved in the evaluation of the thermal relic abundance for different levels of inclusiveness.

We stress also that the inclusion, beyond the $2 \rightarrow 2$ processes, of the $2 \rightarrow 2+W_{f}$ ones, can induce a drastic modification of the cross sections involved into the annihilation processes. In the case of the Lagrangian in Eq. (2.4), for example, $2 \rightarrow 2$ processes are proportional always to $\left|C_{\mathrm{D}}\right|^{2}$ while the $2 \rightarrow 2+W_{f}$ are proportional to $\left|C_{\mathrm{ND}}\right|^{2}$. Such a structural effects are generically included in the coefficient $\mathcal{N} \propto\left|C_{\mathrm{D}} / C_{\mathrm{ND}}\right|^{2}$ that is potentially very large.

\section{An explicit example of IR divergences}

In this Section we analyze the relevance of the cancellation theorems from a more quantitative point of view. For this purpose, we shall retrace the arguments presented in Section 2 considering the effective Lagrangian in Eq. (2.4) as a benchmark model for the explicit calculations. Moreover - in order to catch in the simplest way the relevance of the IR corrections - we use throughout this section the eikonal approximation. This approximation, in fact, controls the long-range dynamic of the soft gauge boson emission thus providing the best-suited tool for the analysis of the IR singularities. In more details, the key point of the eikonal approximation is that the exchange of a soft gauge boson with four-momentum $k$ between two external legs with four-momenta $k_{1,2}$ is described by the following integral

$$
\mathcal{L}_{W}=-\frac{g^{2}}{2} \int \frac{d^{3} k}{(2 \pi)^{3} 2 E_{k}}\left(\frac{k_{1}^{\mu}}{k \cdot k_{1}}-\frac{k_{2}^{\mu}}{k \cdot k_{2}}\right)^{2} .
$$

If the two external legs in Eq. (3.1) are both ultra-relativistic, then the value of the eikonal integral displays the well-known [29,32] double logarithmic behavior $\mathcal{L}_{W}=\left(\alpha_{W} / 4 \pi\right) \ln ^{2}\left(s / m_{W}^{2}\right)$; in this 
kinematical configuration, in fact, both the soft (i.e. when the energy of the emitted gauge boson approaches zero) and the collinear (i.e. when the momentum of the emitted gauge boson becomes parallel to the direction of the emitting particle) singularities are available in the phase space. This simple picture, however, is no longer true for the calculation of the relic density, where we have to deal with a different kinematic involving non-relativistic DM particles $\left(v^{2} \sim 1 / 4\right.$, being $v$ the DM relative velocity). In this case the exchange of a gauge boson in the initial state produces the eikonal factor

$$
\mathcal{L}_{W}=\frac{\alpha_{W}}{4 \pi} \frac{4}{3} v^{2} \ln \frac{M_{1}^{2}}{m_{W}^{2}} .
$$

The resulting single logarithm in Eq. (3.2) is related to the residual soft singularity, while the collinear emission is kinematically forbidden. The interested reader can find more details about this point in Appendix A. Notice that the result in Eq. (3.2) is proportional to the DM relative velocity $v$ because a particle at rest does not emit.

Let us now start our discussion about the cancellation theorems elaborating in more details the annihilation processes described by Eq. (2.2). In particular we first analyze the situation in which the average over the initial flavors is not performed, leading to the violation of the BN theorem $[29,30,34]$. The annihilation of DM particles in the early universe analyzed in this paper represents a completely different physical situation; it is interesting, as a consequence, to better investigate how the BN violation manifests itself in this context. As explained in Section 2, the average over the initial flavors is not required whenever the thermal history of the decoupling prevents the inclusion of the co-annihilation processes. In order to match with the formalism elaborated in [29,34], we first define the following inclusive cross sections

$$
\begin{aligned}
\sigma_{0+} & \equiv \sigma\left(\chi_{0} \chi^{+} \rightarrow f_{1} \bar{f}_{2}\right), \\
\sigma_{0-} & \equiv \sigma\left(\chi_{0} \chi^{-} \rightarrow f_{2} \bar{f}_{1}\right), \\
\sigma_{+-} & \equiv \sigma\left(\chi^{+} \chi^{-} \rightarrow f_{1} \bar{f}_{1}\right)+\sigma\left(\chi^{+} \chi^{-} \rightarrow f_{2} \bar{f}_{2}\right), \\
\sigma_{00} & \equiv \sigma\left(\chi_{0} \chi_{0} \rightarrow f_{1} \bar{f}_{1}\right)+\sigma\left(\chi_{0} \chi_{0} \rightarrow f_{2} \bar{f}_{2}\right),
\end{aligned}
$$

with $\chi_{0} \equiv \chi^{3}, \chi^{ \pm} \equiv\left(\chi^{1} \mp i \chi^{2}\right) / \sqrt{2}$. Using Eq. (2.4) we find

$$
\begin{aligned}
\sigma_{0 \pm}^{\mathrm{H}} & =\frac{C_{\mathrm{ND}}^{2} N_{C}^{f} s\left[48 M_{1}^{2}+s\left(12+v^{2}\right)\right]}{6 \pi \Lambda^{4} \sqrt{s^{2}\left(4+v^{2}\right)^{2}-256 M_{1}^{4}}} \\
\sigma_{+-}^{\mathrm{H}} & =\frac{N_{C}^{f} s\left\{C_{\mathrm{D}}^{2}\left[s\left(12+v^{2}\right)-48 M_{1}^{2}\right]+C_{\mathrm{ND}}^{2}\left[48 M_{1}^{2}+s\left(12+v^{2}\right)\right]\right\}}{6 \pi \Lambda^{4} \sqrt{s^{2}\left(4+v^{2}\right)^{2}-256 M_{1}^{4}}} \\
\sigma_{00}^{\mathrm{H}} & =\frac{C_{\mathrm{D}}^{2} N_{C}^{f} s\left[s\left(12+v^{2}\right)-48 M_{1}^{2}\right]}{6 \pi \Lambda^{4} \sqrt{s^{2}\left(4+v^{2}\right)^{2}-256 M_{1}^{4}}}
\end{aligned}
$$

where the superscript ${ }^{\mathrm{H}}$ indicates that these inclusive hard cross sections are evaluated at the tree level while for the square of the total energy in the center of mass frame we have $s=4 M_{1}^{2} /\left(1-v^{2} / 4\right)$. The color factor $N_{C}^{f}$ is equal to 3 (1) for final state involving quarks (leptons). Considering the 
expansion $v \sigma_{i j}^{\mathrm{H}}=a_{i j}+b_{i j} v^{2}+\mathcal{O}\left(v^{4}\right)$, and dropping terms $\mathcal{O}\left(v^{4}\right)$, we find

$$
\begin{aligned}
v \sigma_{0 \pm}^{\mathrm{H}} & =\frac{2 M_{1}^{2} C_{\mathrm{ND}}^{2} N_{C}^{f}}{\pi \Lambda^{4}}\left(2+\frac{v^{2}}{3}\right) \\
v \sigma_{+-}^{\mathrm{H}} & =\frac{2 M_{1}^{2} N_{C}^{f}}{\pi \Lambda^{4}}\left[2 C_{\mathrm{ND}}^{2}+\frac{v^{2}\left(C_{\mathrm{D}}+C_{\mathrm{ND}}^{2}\right)}{3}\right] \\
v \sigma_{00}^{\mathrm{H}} & =\frac{2 M_{1}^{2} C_{\mathrm{D}}^{2} N_{C} v^{2}}{3 \pi \Lambda^{4}}
\end{aligned}
$$

where in particular the p-wave behavior of $v \sigma_{00}^{\mathrm{H}}$ becomes evident. Notice that these cross sections satisfy the sum rules dictated by the $\mathrm{SU}(2)_{L}$ invariance [34], namely $\sigma_{++}=\sigma_{--}, \sigma_{0+}=\sigma_{0-}$, $\sigma_{00}=\sigma_{++}+\sigma_{+-}-\sigma_{0+}$, where in this particular case $\sigma_{ \pm \pm}=0$. The inclusion of the one-loop electroweak IR corrections changes the tree level cross section $\sigma_{00}^{\mathrm{H}}$ into its "dressed" form [34]

$$
\sigma_{00}=\sigma_{00}^{\mathrm{H}}-4\left(\sigma_{+-}^{\mathrm{H}}-2 \sigma_{3+}^{\mathrm{H}}\right) \mathcal{L}_{W},
$$

with $\mathcal{L}_{W}$ given in Eq. (3.2). This extremely concise formula contains the sum of real and virtual IR corrections to the DM annihilation cross section, and the logarithmic dependence is the trademark of the BN violation. Defining $\Delta \sigma_{00} \equiv \sigma_{00}-\sigma_{00}^{\mathrm{H}}$, and using for the DM relative velocity at the freeze-out the value $v^{2} \sim 1 / 4$ we find the relative correction

$$
\frac{\Delta \sigma_{00}}{\sigma_{00}^{\mathrm{H}}}=\left(\frac{\sigma_{0+}^{\mathrm{H}}}{\sigma_{00}^{\mathrm{H}}}-1\right) \frac{\alpha_{W}}{4 \pi} \frac{4}{3} \ln \frac{M_{1}^{2}}{m_{W}^{2}} .
$$

The BN-violating IR corrections always enhance the tree level cross section $\sigma_{00}^{\mathrm{H}}$ if $\sigma_{0+}^{\mathrm{H}} \geqslant \sigma_{00}^{\mathrm{H}}$; considering the analytic expressions given in Eqs. (3.10,3.12), this remains true as long as $C_{\mathrm{ND}} / C_{\mathrm{D}} \gtrsim 0.2$. We show the ratio $\Delta \sigma_{00} / \sigma_{00}^{\mathrm{H}}$ in Fig. 1 as a function of the DM mass considering three different benchmark values for the coefficients of the effective Lagrangian in Eq. (2.4), $C_{\mathrm{ND}} / C_{\mathrm{D}}=1,4,10$. For a $\mathrm{TeV}$-scale DM particle the effect of the BN-violating IR corrections can easily reach the $50 \%$ level. Even in this simple example, therefore, we can fully appreciate the considerable impact of these corrections. Bearing in mind that the one-loop virtual corrections always lead to a suppression of the cross section, in fact, the inclusion of the real emission processes drastically overturns this scenario: the net effect of the radiative corrections becomes positive, increasing the DM annihilation rate. This shows that the violation of the $\mathrm{BN}$ theorem may lead to corrections much larger than those analyzed, for instance, in Refs. [18-22] for the supersymmetric DM and where corrections to the DM density of at most $10 \%$ were found.

Given the astonishing precision reached for the experimental measurement of the physical density of cold DM in the universe $\left(\Omega_{\mathrm{DM}} h^{2}=0.1199 \pm 0.0027\right.$ [1] $)$, a careful treatment of these corrections is mandatory. Such corrections have to be added to those recently pointed out in Ref. [23], originating from a proper consideration of the temperature dependence of the number of degrees of freedom, which also can be of the order of $60 \%$.

We stress once again that, beyond the toy model used for illustrative purposes in this paper, the only ingredient needed for the computation of these corrections is the isospin quantum number of the DM multiplet together with the eikonal integral in Eq. (3.2).

Let us now move to discuss in more details the situation in which the co-annihilation processes become important. In this case the necessity to perform the average over the initial flavors washes 


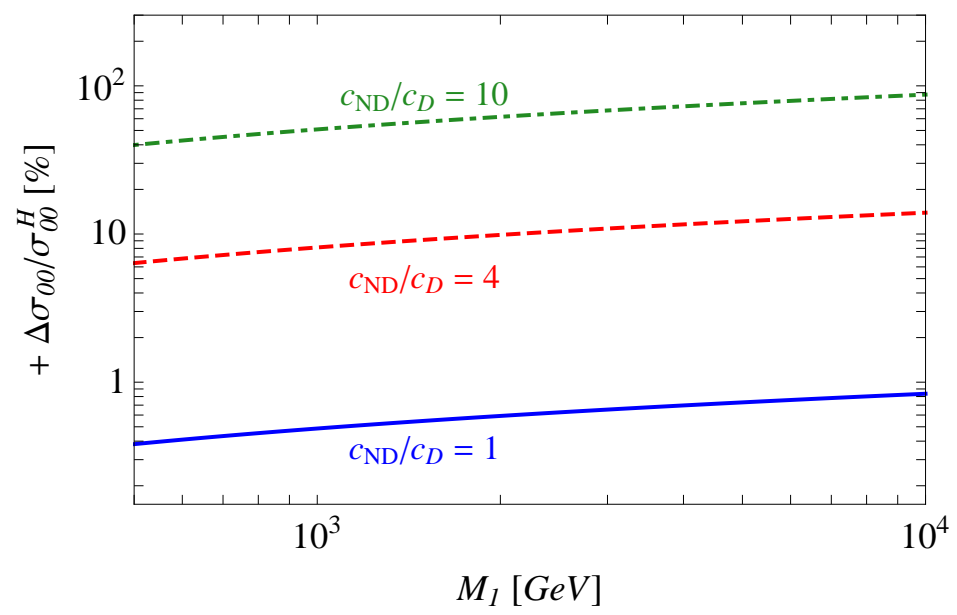

Figure 1: BN-violating IR corrections to the annihilation cross section $\sigma_{00}$ defined in Eq. (3.6). We plot the relative correction $\Delta \sigma_{00} / \sigma_{00}^{\mathrm{H}}$ in Eq. (3.14) as a function of the DM mass for three different values of the ratio $C_{\mathrm{ND}} / C_{\mathrm{D}}$.

out the logarithm in Eq. (3.13) as a consequence of the BN theorem. Nevertheless, as explained in the previous Section, a residual logarithmic correction proportional to the mass splitting $\Delta_{a}$ survives. This correction, whose magnitude is estimated in Eq. (2.11), originates from the different Boltzmann weights that affect each component of the DM multiplet in the thermal bath, and formally violates the BN theorem.

Moreover, the isospin-breaking terms that lift the degeneracy in the DM multiplet are by themselves a possible source of BN violation. Besides the corrections of dynamical type in Eq. (2.11), therefore, one should include also these isospin-breaking effects. However, it is possible to show that the latter are sub-leading if compared to the former. To be convinced, one can account for the mass splitting effects introducing the following improved eikonal current $J_{\text {eik, } \Delta}^{\mu}$ (see [33])

$$
J_{\text {eik }}^{\mu}=g \frac{k^{\mu}}{p \cdot k} \quad \Longrightarrow \quad J_{\text {eik, } \Delta}^{\mu} \equiv g \frac{\left(2 k^{\mu}+M_{1} \Delta_{a} \gamma^{\mu}\right)}{2 p \cdot k}
$$

describing the emission of a soft gauge boson with four-momentum $k$ from an initial DM particle with four-momentum $p$. An explicit calculation shows that the residual logarithmic corrections arise from the diagrams involving only emission from the initial states, ${ }^{2}$ and that their explicit form has the following structure

$$
\mathcal{O}\left(\Delta_{a}^{2} \cdot \mathcal{N} \cdot \frac{\alpha_{W}}{4 \pi} \cdot \ln \frac{M_{1}^{2}}{m_{W}^{2}}\right)
$$

thus producing a sub-dominant effect if compared to the thermal correction in Eq. (2.11).

Let us now close our discussion with a final comment. Considering the annihilation of DM particles, the Sommerfeld enhancement might play a relevant role. In a nutshell the Sommerfeld enhancement is a non-relativistic effect generated by the exchange of light force carriers between

\footnotetext{
${ }^{2}$ In particular we find that the corrections proportional to the mass splitting coming from the interference between initial and final legs cancel out.
} 
the incoming DM particles, resulting in the possibility to form a resonant bound state. Using a non-relativistic resummation technique, it has been shown [35] that this enhancement can affect also the calculation of the relic abundance, leading in some cases to a reduction of $50 \%$ (see also [10,36]). However, relying on the creation of a bound state, the Sommerfeld enhancement must fulfill two special conditions in order to reach such large values. Describing the exchange of massive W bosons with a Yukawa potential, ${ }^{3}$ the first condition is that the range of this interaction is larger than the typical Bohr radius of the system consisting of the two DM incoming particles. This request is achieved whenever $\alpha_{W} M_{1} \gtrsim m_{W}$, namely, being $\alpha_{W} \approx 0.03$, for a TeV-scale DM particle. More importantly, the characteristic energy of the interaction must be larger that the kinetic DM energy. This second prerogative leads to the condition $v \lesssim \alpha_{W}$. This inequality can be easily satisfied in the present-day annihilations $\left(v \sim 10^{-3}\right)$, but it seems very unlikely in the early universe $(v \sim 1 / 2)$. The large corrections quoted above, in fact, can happen only in presence of a state slightly heavier than the DM particle. This state can therefore be produced by the incoming DM particles with almost zero velocity, allowing the possibility to generate the Sommerfeld enhancement. More quantitatively, this condition reads $2 \Delta_{a} \lesssim \alpha_{W}^{2}+v^{2} / 4$, where the r.h.s. follows from the sum of the characteristic Bohr energy of the potential and the collision energy of the two DM particles. Using $v \sim 1 / 2$ we have $\Delta_{a} \lesssim 1 / 30$. As noticed in Section 2.2, the reader should also keep in mind that, being a virtual effect, the Sommerfeld enhancement is always proportional to $\left|C_{\mathrm{D}}\right|^{2}$. Notice also that in the case of scalar DM, the operators controlling the mass splitting within the multiplet are generically of lower dimension than for fermion DM, so one expects $\Delta_{a}$ to be larger for scalar DM than for fermion DM. All in all, a complementarity between the Sommerfeld enhancement and the IR corrections arises. On the one hand, in presence of a small mass splitting between the component of the DM multiplet, the IR corrections are screened by the BN theorem thus affecting the Sommerfeld enhancement only with the residual BN-violating log in Eq. (2.11). On the other hand, we argue that in presence of a large mass splitting the BN-violating IR corrections described in this paper will be the dominant effect.

\section{Conclusions}

In this paper we have discussed the important role of EW corrections for the calculation of the DM relic abundance. In fact, the cancellation theorems which prevent log corrections to appear when computing annihilation cross sections with the inclusion of gauge boson emission, may be evaded.

In the situation described as follows

- the DM particle is the electrically neutral component of a $\mathrm{SU}(2)_{L}$ mutliplet, whose components are not degenerate in mass;

- the DM mass $M_{1}$ is much heavier than the weak scale $M_{1} \gg m_{W}$;

- the relative mass splitting within the multiplet is sufficiently large $\Delta_{a}=\left(M_{a}-M_{1}\right) / M_{1} \gtrsim 1 / 25$

we have found that the cancellation theorems are not operative and there are potentially large corrections to the DM relic abundance.

\footnotetext{
${ }^{3}$ In order to allow a clear and immediate comparison with the situation analyzed in this paper, we focus our discussion on the case in which the Sommerfeld enhancement is primed by the exchange of SM gauge bosons rather than new dark mediators, see Ref. [37].
} 
For instance, for a $\mathrm{TeV}$-scale $\mathrm{DM}$ particle the effect of the BN-violating IR corrections can easily reach an enhancement of the $50 \%$ level (see Fig. 1). These corrections have to be added to those due to the entropy changes not considered in this work, e.g. due to the QCD transition from deconfinement to confinement, mass-generation above the electroweak scale, and other possible transitions recently analyzed in Ref. [23], in which the change in the number of degrees of freedom give corrections that can be of the same order of the ones considered here. Therefore, in the era of precision cosmology where the energy density of cold DM in the universe is measured at the percent level, reliable calculations of the DM relic abundance need to take these effects into account.

\section{Acknowledgments}

ADS acknowledges partial support from the European Union FP7 ITN INVISIBLES (Marie Curie Actions, PITN-GA-2011-289442). The work of AU is supported by the ERC Advanced Grant $\mathrm{n}^{\circ}$ 267985, "Electroweak Symmetry Breaking, Flavour and Dark Matter: One Solution for Three Mysteries" (DaMeSyFla). The work of AU is dedicated to Francesco Caracciolo.

\section{A Cancellation of divergences for mass-degenerate states}

In this Appendix we explicitly work out the calculation of the cancellation of the collinear divergent logarithms, to show how the cancellation theorems work in the case where the DM belongs to a multiplet whose states are degenerate in mass. For simplicity, we consider only the non diagonal term in the Lagrangian of Eq. (2.4):

$$
\mathscr{L}_{\mathrm{eff}, \mathrm{ND}}=i \frac{C_{\mathrm{ND}}}{\Lambda^{2}} \epsilon_{a b c}\left(\bar{L} \gamma_{\mu} P_{L} \sigma^{c} L\right)\left(\bar{\chi}^{a} \gamma^{\mu} \chi^{b}\right)
$$

Collinear divergences arise when the scalar product $k \cdot p$ goes to 0 , where $k$ is the momentum of the $W$ emitted or exchanged in the loop, and $p$ is the momentum of one of the two final state leptons. In the non-relativistic limit for the initial state DM particles we expect no collinear divergence. Indeed, if $k_{1}$ is the momentum of the DM particle and $M$ is the common mass of the DM multiplet, we have

$$
k_{1}^{\mu} \simeq\left(M, 0,0, k_{1}\right), \quad k^{\mu} \simeq(k, k \sin \theta, 0, k \cos \theta)
$$

with $M \gg k_{1}, k$. The scalar product is then

$$
k_{1} \cdot k \simeq M k+k_{1} k \cos \theta
$$

that if $k \neq 0$ is non zero for every value of the angle $\theta$.

For what concerns the emission of a $W$ in the final state, we already know that the divergences coming from this effect are cancelled by those coming from the exchange of a $W$ between the two final state legs. Since, as we showed, there is no collinear divergence from initial state radiation, the

only non trivial point is the cancellation of divergences in the interference between initial and final 


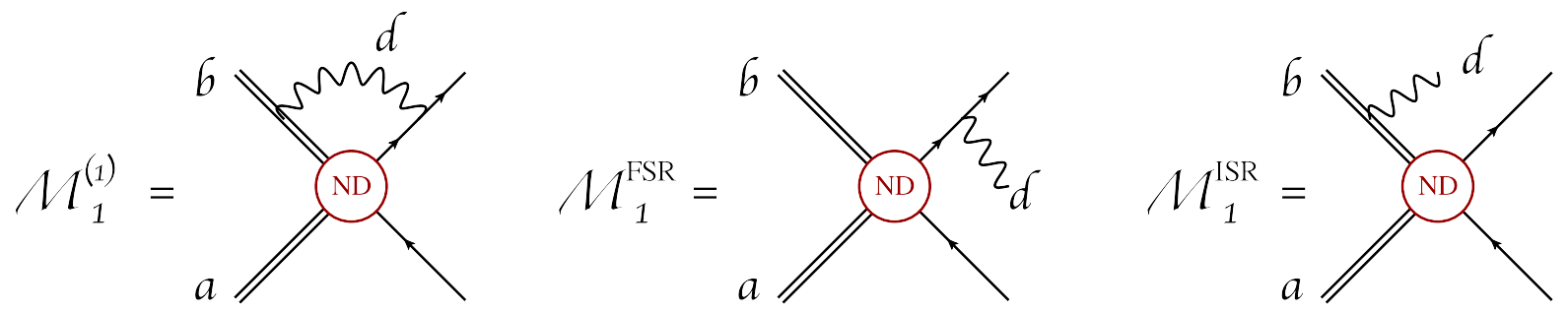

Figure 2: Feynman diagrams corresponding to the scattering amplitudes in Eqs. (A.4-A.6).

state radiation. To do this, we define the three amplitudes

$$
\begin{aligned}
\mathcal{M}_{1}^{(1)} \equiv & \frac{g^{2} C_{\mathrm{ND}}}{2 \Lambda^{2}} \epsilon_{a e c} \epsilon_{b d c} \int \frac{\mathrm{d}^{4} k}{(2 \pi)^{4}}\left\{\frac{\left(g_{\alpha \beta}-k_{\alpha} k_{\beta} / m^{2}\right)}{\left(k^{2}-m^{2}\right)} \bar{v}\left(k_{2}\right) \gamma^{\mu} \frac{\left(\not k_{1}-\not k+M\right)}{\left[\left(k_{1}-k\right)^{2}-M^{2}\right]} \gamma^{\alpha} u\left(k_{1}\right)\right. \\
& \left.\times \bar{u}\left(p_{1}\right) \gamma^{\beta} \frac{\left(\not p_{1}-\not k\right)}{\left(-p_{1} \cdot k+m^{2}\right)} \gamma_{\mu} \sigma^{d} \sigma^{e} P_{L} v\left(p_{2}\right)\right\} \\
\mathcal{M}_{1}^{\mathrm{ISR}} \equiv & -\frac{i g C_{\mathrm{ND}}}{\Lambda^{2}} \epsilon_{a c e} \epsilon_{c d b} \bar{v}\left(k_{2}\right) \gamma^{\mu} \frac{\left(\not k_{1}-\not k+M\right)}{\left[\left(k_{1}-k\right)^{2}-M^{2}\right]} \notin^{*}(k) u\left(k_{1}\right) \bar{u}\left(p_{1}\right) \gamma_{\mu} P_{L} \sigma^{e} v\left(p_{2}\right) \\
\mathcal{M}_{1}^{\mathrm{FSR}} \equiv & \frac{g C_{\mathrm{ND}}}{2 \Lambda^{2}} \bar{v}\left(k_{2}\right) \gamma^{\mu} u\left(k_{1}\right) \bar{u}\left(p_{1}\right) \notin^{*}(k) \frac{\left(\not p_{1}+\not k\right)}{\left(2 p_{1} \cdot k+m^{2}\right)} \gamma_{\mu}\left[\epsilon_{a b d}+i \delta_{b d} \sigma^{a}-i \delta_{a d} \sigma^{b}\right] P_{L} v\left(p_{2}\right)
\end{aligned}
$$

where $m$ is the $W$ mass. The corresponding Feynman diagrams are shown in Fig. 2. Because of the Lorentz structure of the diagrams, to show that the cancellation holds, it is sufficient to show that

$$
\mathcal{M}^{(0)} \cdot\left(\mathcal{M}_{1}^{(1)}\right)^{*}+\mathcal{M}_{1}^{\mathrm{FSR}} \cdot\left(\mathcal{M}_{1}^{\mathrm{ISR}}\right)^{*}
$$

has no logarithmic collinear divergences. The divergences coming from the other similar diagrams will cancel in the same way. In the rest of this appendix we compute separately the two terms using the collinear approximation and show that their sum is indeed finite.

\section{A.1 The amplitude of the $2 \rightarrow 3$ process}

The amplitude of the $2 \rightarrow 3$ process reads

$$
\begin{aligned}
\mathcal{M}_{1}^{\mathrm{FSR}} \cdot\left(\mathcal{M}_{1}^{\mathrm{ISR}}\right)^{*} & =-2 i g^{2} \frac{C_{\mathrm{ND}}^{2}}{\Lambda^{4}} \epsilon_{\alpha}^{*} \epsilon_{\beta} \bar{v}\left(k_{2}\right) \gamma^{\mu} u\left(k_{1}\right) \\
& \times \bar{u}\left(p_{1}\right) \gamma^{\alpha} \frac{\not p_{1}+\not k}{2 p_{1} \cdot k+m^{2}} \gamma_{\mu}\left[\epsilon_{a b d}+i \epsilon_{a b f} \epsilon_{g d f} \sigma^{g}\right] P_{L} v\left(p_{2}\right) \\
& \times \epsilon_{a e c} \epsilon_{b d c} \bar{v}\left(p_{2}\right) \sigma^{e} \gamma_{\nu} P_{L} u\left(p_{1}\right) \bar{u}\left(k_{1}\right) \gamma^{\beta} \frac{\not k_{1}-\not k+M}{\left(k_{1}-k\right)^{2}-M^{2}} \gamma^{\nu} v\left(k_{2}\right) .
\end{aligned}
$$


The $\mathrm{SU}(2)_{L}$ indices give

$$
\begin{aligned}
\sum_{c} \epsilon_{a b d} \epsilon_{a e c} \epsilon_{b d c} & =\epsilon_{a b d}\left(\delta_{a b} \delta_{d e}-\delta_{a d} \delta_{b e}\right)=0 \\
\sum_{c, e, d, f} \epsilon_{a b f} \epsilon_{g d f} \epsilon_{a e c} \epsilon_{b d c} \sigma^{g} \sigma^{e} & =\left(\delta_{a g} \delta_{b d}-\delta_{a d} \delta_{b g}\right)\left(\delta_{a b} \delta_{d e}-\delta_{a d} \delta_{b e}\right) \sigma^{g} \sigma^{e} \\
& =\delta_{a b} \delta_{b d} \sigma^{a} \sigma^{d}-\delta_{a b} \delta_{a d} \sigma^{b} \sigma^{d}-\delta_{a d} \delta_{b d} \sigma^{a} \sigma^{d}+\delta_{a d} \sigma^{b} \sigma^{b} \\
& =-\delta_{a d} \delta_{b d} \sigma^{a} \sigma^{d}+\delta_{a d} \sigma^{b} \sigma^{b}=\left\{\begin{array}{l}
0 \text { when } a=b=d \\
1 \text { when } a=d \neq b
\end{array}\right.
\end{aligned}
$$

so that (A.8) does not vanish only for $a=d \neq b$, and in this case we obtain

$$
\begin{aligned}
\mathcal{M}_{1}^{\mathrm{FSR}} \cdot\left(\mathcal{M}_{1}^{\mathrm{ISR}}\right)^{*} & =2 g^{2} \frac{C_{\mathrm{ND}}^{2}}{\Lambda^{4}} \epsilon_{\alpha}^{*} \epsilon_{\beta} \bar{v}\left(k_{2}\right) \gamma^{\mu} u\left(k_{1}\right) \bar{u}\left(p_{1}\right) \gamma^{\alpha} \frac{\not p 1+\not k}{2 p_{1} \cdot k+m^{2}} \gamma_{\mu} P_{L} v\left(p_{2}\right) \\
& \times \bar{v}\left(p_{2}\right) \gamma_{\nu} P_{L} u\left(p_{1}\right) \bar{u}\left(k_{1}\right) \gamma^{\beta} \frac{\not k_{1}-\not k+M}{\left(k_{1}-k\right)^{2}-M^{2}} \gamma^{\nu} v\left(k_{2}\right) .
\end{aligned}
$$

Summing over spin and polarization states we get

$$
\begin{aligned}
\left\langle\mathcal{M}_{1}^{\mathrm{FSR}} \cdot\left(\mathcal{M}_{1}^{\mathrm{ISR}}\right)^{*}\right\rangle & =-\frac{g^{2}}{2} \frac{C_{\mathrm{ND}}^{2}}{\Lambda^{4}} \frac{1}{2 p_{1} \cdot k+m^{2}} \frac{1}{\left(k_{1}-k\right)^{2}-M^{2}}\left(g_{\alpha \beta}-\frac{k_{\alpha} k_{\beta}}{m^{2}}\right) \\
& \times \operatorname{tr}\left[\gamma^{\mu}\left(\not k_{1}+M\right) \gamma^{\beta}\left(\not k_{1}-\not k+M\right) \gamma^{\nu}\left(\not k_{2}-M\right)\right] \\
& \times \operatorname{tr}\left[\not p_{1} \gamma^{\alpha}\left(\not p_{1}+\not k\right) \gamma_{\mu} \not p_{2} \gamma_{\nu} P_{L}\right] .
\end{aligned}
$$

To compute the cross section we have to integrate Eq. (A.12) with the measure

$$
\frac{\mathrm{d}^{3} p_{1}}{(2 \pi)^{3} 2 E_{1}} \frac{\mathrm{d}^{3} p_{2}}{(2 \pi)^{3} 2 E_{2}} \frac{\mathrm{d}^{3} k}{(2 \pi)^{3} 2 E_{k}} \delta^{4}\left(P_{\text {in }}-p_{1}-p_{2}-k\right),
$$

where $P_{\text {in }}$ is the total momentum of the initial state. We want to factorize the $\mathrm{d}^{3} k$ integral using the collinear approximation. In order to do so, we multiply the measure in Eq. (A.13) by a factor of $1=\int \mathrm{d}^{4} p \delta^{4}\left(p-p_{1}-k\right)$, so that we have

$$
\int \frac{\mathrm{d}^{3} p_{2}}{(2 \pi)^{3} 2 E_{2}} \frac{\mathrm{d}^{3} p_{1}}{(2 \pi)^{3} 2 E_{1}} \frac{\mathrm{d}^{3} k}{(2 \pi)^{3} 2 E_{k}}\left[\int \mathrm{d} p^{0} \delta\left(p^{0}-p_{1}^{0}-k^{0}\right) \mathrm{d}^{3} p \delta^{3}\left(\vec{p}-\vec{p}_{1}-\vec{k}\right)\right] \delta^{4}\left(P_{\text {in }}-p_{1}-p_{2}-k\right) .
$$

Now we integrate over $\mathrm{d} p^{0}$ and over $\mathrm{d}^{3} p_{1}$ using the first two Dirac deltas to get

$$
\int \frac{\mathrm{d}^{3} p_{2}}{(2 \pi)^{3} 2 E_{2}} \frac{\mathrm{d}^{3} k}{(2 \pi)^{3} 2 E_{k}} \frac{\mathrm{d}^{3} p}{(2 \pi)^{3} 2|\vec{p}-\vec{k}|} \delta^{4}\left(P_{\text {in }}-p_{1}-p_{2}-k\right)
$$

Finally using the collinear approximation $\vec{k} \approx x \vec{p}$ we obtain the factorized measure

$$
\int \frac{\mathrm{d}^{3} p}{(2 \pi)^{3} 2 E} \frac{\mathrm{d}^{3} p_{2}}{(2 \pi)^{3} 2 E_{2}} \delta^{4}\left(P_{\text {in }}-p-p_{2}\right) \frac{\mathrm{d}^{3} k}{(2 \pi)^{3} 2 E_{k}}\left(\frac{1}{1-x}\right) .
$$

The $\mathrm{d}^{3} k$ integral can be written in spherical coordinates, using the collinear approximation, as

$$
\int \frac{\mathrm{d}^{3} k}{(2 \pi)^{3} 2 E_{k}}=\int \frac{|\vec{k}|^{2} \mathrm{~d} k \sin \theta \mathrm{d} \theta \mathrm{d} \phi}{(2 \pi)^{3} 2 E_{k}} \approx \frac{1}{2} \int_{0}^{1} \mathrm{~d} x x \int_{-1}^{+1} \mathrm{~d}(\cos \theta) \int_{0}^{2 \pi} \mathrm{d} \phi .
$$


In the collinear limit the traces become

$$
(1-x)\left(g_{\alpha \beta}-x^{2} \frac{p_{\alpha} p_{\beta}}{m^{2}}\right) \operatorname{tr}\left[\gamma^{\mu}\left(\not k_{1}+M\right) \gamma^{\beta}\left(\not k_{1}-x \not p+M\right) \gamma^{\nu}\left(\not k_{2}-M\right)\right] \operatorname{tr}\left[\not p \gamma^{\alpha}(\not p) \gamma_{\mu} \not p_{2} \gamma_{\nu} P_{L}\right],
$$

which, using $p^{2}=0$, reduces to

$$
64(1-x) k_{1} \cdot p\left[\left(k_{1} \cdot p\right)\left(k_{2} \cdot p_{2}\right)+\left(k_{1} \cdot p_{2}\right)\left(k_{2} \cdot p\right)+M^{2} p \cdot p_{2}\right] .
$$

In the non-relativistic limit for the two DM particles, we can take $k_{1}=k_{2}=(M, 0,0,0)$. Then $p=(M, 0,0, M)$ and $p_{2}=(M, 0,0,-M)$, and Eq. (A.19) gives simply $256(1-x) M^{6}$. We have then

$$
\begin{aligned}
\left\langle\mathcal{M}_{1}^{\mathrm{FSR}} \cdot\left(\mathcal{M}_{1}^{\mathrm{ISR}}\right)^{*}\right\rangle & \leadsto-16 g^{2} \frac{C_{\mathrm{ND}}^{2}}{\Lambda^{4}} \frac{M^{8}}{(2 \pi)^{3}} \\
& \times \int_{0}^{2 \pi} \mathrm{d} \phi \int_{-1}^{+1} \mathrm{~d}(\cos \theta) \int_{0}^{1} \mathrm{~d} x \frac{x}{\left(2 p_{1} \cdot k+m^{2}\right)\left[\left(k_{1}-k\right)^{2}-M^{2}\right]},
\end{aligned}
$$

where with the symbol $\leadsto$ we mean that we consider only the collinear divergent part.

The three vectors $k, p_{1}$ and $p_{2}$ can be parametrized as

$$
\begin{aligned}
k & =\left(x E, \sqrt{x^{2} E^{2}-m^{2}} \sin \theta \cos \phi, \sqrt{x^{2} E^{2}-m^{2}} \sin \theta \sin \phi, \sqrt{x^{2} E^{2}-m^{2}} \cos \theta\right), \\
p_{1} & =\left(y E,-\sqrt{x^{2} E^{2}-m^{2}} \sin \theta \cos \phi,-\sqrt{x^{2} E^{2}-m^{2}} \sin \theta \sin \phi, \sqrt{y^{2} E^{2}-\left(x^{2} E^{2}-m^{2}\right) \sin ^{2} \theta}\right),
\end{aligned}
$$

$$
p_{2}=(z E, 0,0,-z E) .
$$

Ignoring the $W$ mass, at order $\theta^{2}$ the scalar product $k \cdot p_{1}$ is equal to

$$
k \cdot p_{1} \simeq \frac{x}{2(1-x)} E^{2} \theta^{2} .
$$

We substitute in the integral the expressions

$$
\begin{aligned}
2 p_{1} \cdot k+m^{2} & \longrightarrow \frac{x}{1-x} M^{2} \theta^{2}+m^{2}, \\
\left(k_{1}-k\right)^{2}-M^{2} & \longrightarrow 2 x M^{2}+m^{2}, \\
\int \mathrm{d}(\cos \theta) & \longrightarrow \int \theta \mathrm{d} \theta,
\end{aligned}
$$

so that

$$
\left\langle\mathcal{M}_{1}^{\mathrm{FSR}} \cdot\left(\mathcal{M}_{1}^{\mathrm{ISR}}\right)^{*}\right\rangle \leadsto-64 g^{2} \frac{C_{\mathrm{ND}}^{2}}{\Lambda^{4}} \frac{M^{8}}{(2 \pi)^{2}} \int_{0}^{1} \mathrm{~d} x \int_{0}^{2 \pi} \mathrm{d} \theta \frac{\theta x}{\left(\frac{x}{1-x} M^{2} \theta^{2}+m^{2}\right)\left[2 x M^{2}+m^{2}\right]} .
$$

The $\mathrm{d} \theta$ integral is

$$
\frac{1}{2} \int_{0}^{2 \pi} \frac{\mathrm{d} \theta^{2}}{\frac{x}{1-x} M^{2} \theta^{2}+m^{2}}=\frac{1-x}{2 x M^{2}} \log \left(1+\pi^{2} \frac{x}{1-x} \frac{M^{2}}{m^{2}}\right)
$$

and therefore, keeping only the collinear log-divergent part, we obtain

$$
\left\langle\mathcal{M}_{1}^{\mathrm{FSR}} \cdot\left(\mathcal{M}_{1}^{\mathrm{ISR}}\right)^{*}\right\rangle \leadsto-32 g^{2} \frac{C_{\mathrm{ND}}^{2}}{\Lambda^{4}} \frac{M^{6}}{(2 \pi)^{2}}\left(\int_{m / M}^{1} \mathrm{~d} x \frac{1-x}{2 x M^{2}+m^{2}}\right) \log \left(\frac{M^{2}}{m^{2}}\right) .
$$




\section{A.2 The amplitude of the $2 \rightarrow 2$ process}

The amplitude of the $2 \rightarrow 2$ process reads

$$
\begin{aligned}
\mathcal{M}^{(0)} \cdot\left(\mathcal{M}_{1}^{(1)}\right)^{*} & =-2 g^{2} \frac{C_{\mathrm{ND}}^{2}}{\Lambda^{4}} \epsilon_{a b f} \epsilon_{a e c} \epsilon_{b d c} \bar{v}\left(k_{2}\right) \gamma^{\nu} u\left(k_{1}\right) \bar{u}\left(p_{1}\right) \gamma_{\nu} P_{L} \sigma^{f} v\left(p_{2}\right) \\
& \times\left(\int \frac{\mathrm{d}^{4} k}{(2 \pi)^{4}} \frac{g_{\alpha \beta}-\frac{k_{\alpha} k_{\beta}}{m^{2}}}{k^{2}-m^{2}+i \epsilon} \bar{v}\left(k_{2}\right) \gamma^{\mu} \frac{\not k_{1}-\not k+M}{\left(k_{1}-k\right)^{2}-M^{2}+i \epsilon} \gamma^{\alpha} u\left(k_{1}\right)\right. \\
& \left.\times \bar{u}\left(p_{1}\right) \gamma^{\beta} \frac{\not p_{1}-\not k}{\left(p_{1}-k\right)^{2}} \gamma_{\mu} \sigma^{d} \sigma^{e} P_{L} v\left(p_{2}\right)\right)^{*} .
\end{aligned}
$$

In $\mathrm{SU}(2)_{L}$ space we have

$$
\epsilon_{a e c} \epsilon_{b d c} \epsilon_{a b f}=\left(\delta_{a b} \delta_{d e}-\delta_{a d} \delta_{b c}\right) \epsilon_{a b f}=-\delta_{a d} \delta_{b c} \epsilon_{a b f},
$$

that is non-zero only for $a=d \neq b$; therefore we get

$$
\begin{aligned}
\mathcal{M}^{(0)} \cdot\left(\mathcal{M}_{1}^{(1)}\right)^{*} & =+2 g^{2} \frac{C_{\mathrm{ND}}^{2}}{\Lambda^{4}} \epsilon_{a b f} \\
& \times \int \frac{\mathrm{d}^{4} k}{(2 \pi)^{4}}\left(g_{\alpha \beta}-\frac{k_{\alpha} k_{\beta}}{m^{2}}\right) \frac{1}{k^{2}-m^{2}-i \epsilon} \frac{1}{\left(k_{1}-k\right)^{2}-M^{2}-i \epsilon} \frac{1}{\left(p_{1}-k\right)^{2}-i \epsilon} \\
& \times \bar{v}\left(k_{2}\right) \gamma^{\nu} u\left(k_{1}\right) \bar{u}\left(k_{1}\right) \gamma^{\alpha}\left(\not k_{1}-\not k+M\right) \gamma^{\mu} v\left(k_{2}\right) \bar{u}\left(p_{1}\right) \gamma_{\nu} P_{L} \sigma^{f} v\left(p_{2}\right) \\
& \times \bar{v}\left(p_{2}\right) \gamma_{\mu}\left(\not p_{1}-\not k\right) \gamma^{\beta} P_{L} \sigma^{b} \sigma^{a} u\left(p_{1}\right) .
\end{aligned}
$$

Since $a, b$ are different, $\sigma^{b} \sigma^{a}=-i \epsilon_{a b c} \sigma^{c}$. There is only one possible value for $c$ and $f$ must be equal to it. We can therefore write $\sigma^{b} \sigma^{a}$ with $-i \epsilon_{a b f} \sigma^{f}$ (without sum over $f$ ); this multiplied by the other $\epsilon_{a b f}$ gives $\left(\epsilon_{a b f}\right)^{2}=1$. In the $\mathrm{SU}(2)_{L}$ space we have

$$
\bar{v}\left(p_{2}\right)_{i} \sigma_{i j}^{f} u\left(p_{1}\right)_{j} \bar{u}\left(p_{1}\right)_{j} \sigma_{j i}^{f} v\left(p_{2}\right)_{i}, \quad \text { no summation over indices, }
$$

where the value of the indices $i, j$ is fixed by the final state. Since $\sigma_{i j}^{f} \sigma_{j i}^{f}=1$ we can simply forget about the $\sigma$ matrices in Eq. (A.33). We have then (after summing over spin states)

$$
\begin{aligned}
\left\langle\mathcal{M}^{(0)} \cdot\left(\mathcal{M}_{1}^{(1)}\right)^{*}\right\rangle & =-\frac{i}{2} g^{2} \frac{C_{\mathrm{ND}}^{2}}{\Lambda^{4}} \int \frac{\mathrm{d}^{4} k}{(2 \pi)^{4}}\left(g_{\alpha \beta}-\frac{k_{\alpha} k_{\beta}}{m^{2}}\right) \\
& \times \frac{1}{k^{2}-m^{2}-i \epsilon} \frac{1}{\left(k_{1}-k\right)^{2}-M^{2}-i \epsilon} \frac{1}{\left(p_{1}-k\right)^{2}-i \epsilon} \\
& \times \operatorname{tr}\left[\gamma^{\mu}\left(\not k_{1}+M\right) \gamma^{\beta}\left(\not k_{1}-\not k+M\right) \gamma^{\nu}\left(\not k_{2}-M\right)\right] \\
& \times \operatorname{tr}\left[\not p_{1} \gamma_{\nu} \not p_{2} \gamma_{\mu}\left(\not \not_{1}-\not k\right) \gamma^{\beta} P_{L}\right] .
\end{aligned}
$$

We now have to perform the $\mathrm{d} k^{0}$ integral using the Cauchy formula. The three factors in the denominator coming from the three propagators give six poles in the complex plane. Since we are interested only in the part that is divergent for $p_{1} \cdot k \rightarrow 0$, we can consider only the poles coming from the propagator of the $W$, ignoring the others. Closing the contour of integration in the upper or in the lower semi-plane, we get

$$
\int \frac{\mathrm{d}^{4} k}{(2 \pi)^{4}} \frac{1}{k^{2}-m^{2}-i \epsilon} \stackrel{\epsilon \rightarrow 0}{=} i \int \frac{\mathrm{d}^{3} k}{(2 \pi)^{3}} \frac{1}{2 E_{k}},
$$


so that

$$
\begin{aligned}
\left\langle\mathcal{M}^{(0)} \cdot\left(\mathcal{M}_{1}^{(1)}\right)^{*}\right\rangle & \approx \frac{1}{2} g^{2} \frac{C_{\mathrm{ND}}^{2}}{\Lambda^{4}} \int \frac{\mathrm{d}^{3} k}{(2 \pi)^{3}}\left(g_{\alpha \beta}-\frac{k_{\alpha} k_{\beta}}{m^{2}}\right) \frac{1}{2 E_{k}} \frac{1}{\left(k_{1}-k\right)^{2}-M^{2}} \frac{1}{\left(p_{1}-k\right)^{2}} \times \\
& \times \operatorname{tr}\left[\gamma^{\mu}\left(\not k_{1}+M\right) \gamma^{\beta}\left(\not k_{1}-\not k+M\right) \gamma^{\nu}\left(\not k_{2}-M\right)\right] \\
& \times \operatorname{tr}\left[\not \not_{1} \gamma_{\nu} \not \not_{2} \gamma_{\mu}\left(\not \not_{1}-\not k\right) \gamma^{\beta} P_{L}\right] .
\end{aligned}
$$

If we define $x$ as in the previous subsection, in the collinear limit we have $k \approx x p_{1}$ with $p_{1} \approx$ $(E, 0,0, E)$ and $p_{2} \approx(E, 0,0,-E)$ where $2 E$ is the center of mass energy. The traces become then

$$
(1-x)\left(g_{\alpha \beta}-x^{2} \frac{p_{1 \alpha} p_{1 \beta}}{m^{2}}\right) \operatorname{tr}\left[\gamma^{\mu}\left(\not k_{1}+M\right) \gamma^{\beta}\left(\not k_{1}-x \not p_{1}+M\right) \gamma^{\nu}\left(\not k_{2}-M\right)\right] \operatorname{tr}\left[\not p_{1} \gamma^{\alpha}\left(\not p_{1}\right) \gamma_{\mu} \not p_{2} \gamma_{\nu} P_{L}\right]
$$

that is the same expression obtained in the previous subsection. In the non-relativistic limit we get simply $256(1-x) M^{6}$.

The integral in $\mathrm{d}^{3} k$ can be written in spherical coordinates in the same way as before. Notice that, despite the fact that in a loop integral one should integrate over alla momenta up to $+\infty$, here the $\mathrm{d} x$ integral goes from $m / M$ to 1 . Physically this is due to the fact that, integrating around the $W$ propagator poles, we are putting the $W$ on-shell. Then this $W$ behaves like a physical particle, and its momenta cannot be greater than the initial energy. The final result is then

$$
\left\langle\mathcal{M}^{(0)} \cdot\left(\mathcal{M}_{1}^{(1)}\right)^{*}\right\rangle \leadsto 64 g^{2} \frac{C_{\mathrm{ND}}^{2}}{\Lambda^{4}} \frac{M^{8}}{(2 \pi)^{3}} \int_{0}^{2 \pi} \mathrm{d} \phi \int_{-1}^{+1} \mathrm{~d}(\cos \theta) \int_{0}^{1} \mathrm{~d} x \frac{x(1-x)}{\left(2 p_{1} \cdot k+m^{2}\right)\left[\left(k_{1}-k\right)^{2}-M^{2}\right]} .
$$

In function of $x, \theta$ and $\phi, k$ is parametrized as

$$
k=\left(x E, \sqrt{x^{2} E^{2}-m^{2}} \sin \theta \cos \phi, \sqrt{x^{2} E^{2}-m^{2}} \sin \theta \cos \phi, \sqrt{x^{2} E^{2}-m^{2}} \cos \theta\right) .
$$

Ignoring the $W$ mass, at order $\theta^{2}$ the scalar product $k \cdot p_{1}$ becomes

$$
p_{1} \cdot k=\frac{1}{2} x E^{2} \theta^{2}
$$

so that

$$
\left\langle\mathcal{M}^{(0)} \cdot\left(\mathcal{M}_{1}^{(1)}\right)^{*}\right\rangle \leadsto 64 g^{2} \frac{C_{\mathrm{ND}}^{2}}{\Lambda^{4}} \frac{M^{8}}{(2 \pi)^{2}} \int_{m / M}^{1} \mathrm{~d} x \int_{0}^{2 \pi} \mathrm{d} \theta \frac{\theta x(1-x)}{\left(x M^{2} \theta^{2}+m^{2}\right)\left(2 x M^{2}+m^{2}\right)} .
$$

Performing the $\mathrm{d} \theta$ integral we obtain

$$
\left\langle\mathcal{M}^{(0)} \cdot\left(\mathcal{M}_{1}^{(1)}\right)^{*}\right\rangle \leadsto 32 g^{2} \frac{C_{\mathrm{ND}}^{2}}{\Lambda^{4}} \frac{M^{6}}{(2 \pi)^{2}} \int_{m / M}^{1} \mathrm{~d} x\left\{\frac{1-x}{2 x M^{2}+m^{2}} \log \left(1+\pi^{2} x \frac{M^{2}}{m^{2}}\right)\right\} .
$$

The collinear log-divergent part is

$$
\left\langle\mathcal{M}_{1}^{\mathrm{FSR}} \cdot\left(\mathcal{M}_{1}^{\mathrm{ISR}}\right)^{*}\right\rangle \leadsto 32 g^{2} \frac{C_{\mathrm{ND}}^{2}}{\Lambda^{4}} \frac{M^{6}}{(2 \pi)^{2}}\left(\int_{m / M}^{1} \mathrm{~d} x \frac{1-x}{2 x M^{2}+m^{2}}\right) \log \left(\frac{M^{2}}{m^{2}}\right)
$$

that exactly cancels with (A.30). 


\section{References}

[1] P. A. R. Ade et al. [Planck Collaboration], arXiv:1303.5076.

[2] P. Ciafaloni and A. Urbano, Phys. Rev. D 82, 043512 (2010) [arXiv:1001.3950].

[3] P. Ciafaloni, D. Comelli, A. Riotto, F. Sala, A. Strumia and A. Urbano, JCAP 1103, 019 (2011) [arXiv:1009.0224].

[4] N. F. Bell, J. B. Dent, T. D. Jacques and T. J. Weiler, Phys. Rev. D 83, 013001 (2011) [arXiv:1009.2584]; N. F. Bell, J. B. Dent, T. D. Jacques and T. J. Weiler, Phys. Rev. D 84, 103517 (2011) [arXiv:1101.3357].

[5] P. Ciafaloni, M. Cirelli, D. Comelli, A. De Simone, A. Riotto and A. Urbano, JCAP 1106, 018 (2011) [arXiv:1104.2996].

[6] N. F. Bell, J. B. Dent, A. J. Galea, T. D. Jacques, L. M. Krauss and T. J. Weiler, Phys. Lett. B 706, 6 (2011) [arXiv:1104.3823].

[7] K. Cheung, P. -Y. Tseng and T. -C. Yuan, JCAP 1106, 023 (2011) [arXiv:1104.5329].

[8] M. Garny, A. Ibarra and S. Vogl, JCAP 1107, 028 (2011) [arXiv:1105.5367].

[9] P. Ciafaloni, M. Cirelli, D. Comelli, A. De Simone, A. Riotto, A. Urbano, JCAP 1110, 034 (2011) [arXiv:1107.4453].

[10] A. Hryczuk and R. Iengo, JHEP 1201, 163 (2012) [Erratum-ibid. 1206, 137 (2012)] [arXiv:1111.2916].

[11] V. Barger, W. -Y. Keung and D. Marfatia, Phys. Lett. B 707, 385 (2012) [arXiv:1111.4523].

[12] M. Garny, A. Ibarra and S. Vogl, JCAP 1204, 033 (2012) [arXiv:1112.5155].

[13] A. De Simone, J. Phys. Conf. Ser. 375, 012046 (2012) [arXiv:1201.1443].

[14] A. De Simone, A. Monin, A. Thamm and A. Urbano, JCAP 1302, 039 (2013) [arXiv:1301.1486].

[15] L. Bergstrom, Phys. Lett. B 225, 372 (1989).

[16] T. Bringmann, L. Bergstrom and J. Edsjo, JHEP 0801, 049 (2008) [arXiv:0710.3169]; L. Bergstrom, T. Bringmann and J. Edsjo, Phys. Rev. D 78, 103520 (2008) [arXiv:0808.3725].

[17] V. Berezinsky, M. Kachelriess and S. Ostapchenko, Phys. Rev. Lett. 89, 171802 (2002) [hepph/0205218]; C. Barbot and M. Drees, Phys. Lett. B 533, 107 (2002) [hep-ph/0202072]; C. Barbot and M. Drees, Astropart. Phys. 20, 5 (2003) [hep-ph/0211406]; M. Kachelriess and P. D. Serpico, Phys. Rev. D 76, 063516 (2007) [arXiv:0707.0209]; N. F. Bell, J. B. Dent, T. D. Jacques and T. J. Weiler, Phys. Rev. D 78, 083540 (2008) [arXiv:0805.3423]; J. B. Dent, R. J. Scherrer and T. J. Weiler, Phys. Rev. D 78, 063509 (2008) [arXiv:0806.0370]; V. Barger, Y. Gao, W. Y. Keung, D. Marfatia, Phys. Rev. D80, 063537 (2009) [arXiv:0906.3009]; J. F. Fortin, J. Shelton, S. Thomas and Y. Zhao, arXiv:0908.2258; M. Kachelriess, P. D. Serpico and M. A. Solberg, Phys. Rev. D 80, 123533 (2009) [arXiv:0911.0001]. 
[18] N. Baro, F. Boudjema, G. Chalons and S. Hao, Phys. Rev. D 81, 015005 (2010) [arXiv:0910.3293].

[19] B. Herrmann, M. Klasen and K. Kovarik, Phys. Rev. D 80, 085025 (2009) [arXiv:0907.0030].

[20] F. Boudjema, G. Drieu La Rochelle and S. Kulkarni, Phys. Rev. D 84, 116001 (2011) [arXiv:1108.4291].

[21] A. Chatterjee, M. Drees and S. Kulkarni, Phys. Rev. D 86, 105025 (2012) [arXiv:1209.2328].

[22] J. Harz, B. Herrmann, M. Klasen, K. Kovarik and Q. L. Boulc'h, Phys. Rev. D 87, 054031 (2013) [arXiv:1212.524].

[23] G. Steigman, B. Dasgupta and J. F. Beacom, Phys. Rev. D 86, 023506 (2012) [arXiv:1204.3622].

[24] T. D. Lee and M. Nauenberg, Phys. Rev. 133 (1964) B1549. V. Chung, Phys. Rev. 140 (1965) B1110. T. Kinoshita, J. Math. Phys. 3 (1962) 650.

[25] F. Bloch and A. Nordsieck, Phys. Rev. 52 (1937) 54. D. R. Yennie, S. C. Frautschi and H. Suura, Annals Phys. 13 (1961) 379.

[26] T. W. B. Kibble, Phys. Rev. 174 (1968) 1882. T. W. B. Kibble, Phys. Rev. 175 (1968) 1624. P. P. Kulish and L. D. Faddeev, Theor. Math. Phys. 4 (1970) 745 [Teor. Mat. Fiz. 4 (1970) 153].

[27] M. Ciafaloni, Phys. Lett. B 150 (1985) 379. S. Catani, M. Ciafaloni and G. Marchesini, Nucl. Phys. B 264 (1986) 588. M. Ciafaloni, Adv. Ser. Direct. High Energy Phys. 5 (1989) 491.

[28] M. Ciafaloni, P. Ciafaloni and D. Comelli, Phys. Rev. Lett. 87, 211802 (2001) [hep-ph/0103315].

[29] M. Ciafaloni, P. Ciafaloni and D. Comelli, Phys. Rev. Lett. 84, 4810 (2000) [hep-ph/0001142].

[30] M. Ciafaloni, P. Ciafaloni and D. Comelli, Nucl. Phys. B 589, 359 (2000) [hep-ph/0004071]; M. Ciafaloni, P. Ciafaloni and D. Comelli, Nucl. Phys. B 613, 382 (2001) [hep-ph/0103316]; P. Ciafaloni and D. Comelli, JHEP 0609, 055 (2006) [hep-ph/0604070]; P. Ciafaloni and A. Urbano, Phys. Rev. D 81, 085033 (2010) [arXiv:0902.1855];

[31] K. Griest and D. Seckel, Phys. Rev. D 43, 3191 (1991).

[32] P. Ciafaloni and D. Comelli, Phys. Lett. B 446, 278 (1999) [hep-ph/9809321].

[33] M. Ciafaloni, P. Ciafaloni and D. Comelli, JHEP 1003 (2010) 072 [arXiv:0909.1657].

[34] M. Ciafaloni, P. Ciafaloni and D. Comelli, Phys. Lett. B 501, 216 (2001) [hep-ph/0007096].

[35] J. Hisano, S. Matsumoto and M. M. Nojiri, Phys. Rev. D 67, 075014 (2003) [hep-ph/0212022]. J. Hisano, S. Matsumoto, M. Nagai, O. Saito and M. Senami, Phys. Lett. B 646, 34 (2007) [hep-ph/0610249].

[36] A. Hryczuk, R. Iengo and P. Ullio, JHEP 1103, 069 (2011) [arXiv:1010.2172].

[37] N. Arkani-Hamed, D. P. Finkbeiner, T. R. Slatyer and N. Weiner, Phys. Rev. D 79, 015014 (2009) [arXiv:0810.0713]. 